\title{
Little evidence available for arginine and caries prevention
}

\author{
Abstracted from \\ Ástvaldsdóttir Á, Naimi-Akbar A, Davidson T, Brolund A, Lintamo L, Attergren Granath A, Tranæus S, Östlund P. \\ Arginine and Caries Prevention: A Systematic Review. \\ Caries Res 2016; 50: 383-393. doi: 10.1159/000446249. Epub 2016 Jul 12. PubMed PMID: 27403876.
}

Address for correspondence: Álfheiður Ástvaldsdóttir, Department of Dental Medicine, Karolinska Institutet,

PO Box 4064, SE-14104 Huddinge (Sweden). E-mail: Alfheidur.Astvaldsdottir@ki.se

\section{Question: Do arginine-containing dental products prevent or slow progression of caries?}

Data Sources PubMed, the Cochrane Library, Centre for Reviews and Dissemination (CRD) and Embase.

Data abstraction and synthesis Two independent reviewers selected studies, abstracted data and assessed study quality using the GRADE criteria. Meta-analysis was conducted using a random effects model. Study selection Randomised controlled trials including in situ studies. Results Seven studies were included, five conducted in children and two in adults. The two adult studies and one child study were considered to be at high risk of bias. The remaining four child studies were considered to be at moderate risk of bias. These four studies compared 1450ppm fluoride toothpaste with $1.5 \%$ arginine against $1450 \mathrm{ppm}$ fluoride toothpaste as a control. Meta-analysis of three studies showed a positive effect on caries measured using quantitative light-induced fluorescence (QLF) in favour of arginine; mean difference $-4.67,(95 \% \mathrm{Cl},-6.34$ to -3.01$)$. The overall GRADE assessment of this was considered to be very low.

Conclusion At present there is insufficient evidence in support of a caries-preventive effect for the inclusion of arginine in toothpastes. More rigorous studies, and studies which are less dependent on commercial interests, are required.

\section{Commentary}

While the introduction of fluoridated toothpaste has helped contribute to important reductions in dental caries, caries remains a significant public health problem worldwide. The amino acid arginine naturally occurs in a range of food products and saliva and metabolises to form ammonia-like substances that increase the $\mathrm{pH}$ of saliva, which can neutralise the acidification effects of sugar metabolism. Research into anticaries effects of arginine has been underway for several decades and arginine has recently been introduced as an additive to toothpaste and other oral healthcare products.

The aim of this review was to assess the evidence on the use of arginine-containing dental care products to prevent the development of new caries lesions and the progression of existing lesions. The review was conducted for the Swedish Agency for Health Technology Assessment and Assessment of Social Services (http://www.sbu.se/en/) which, like NICE (The National Institute for Health and Care Excellence), uses a robust methodological approach. An extensive search and thorough quality assessment of the available studies was undertaken. However, only a small number of studies met the strict inclusion criteria and the overall quality was considered to be low. Only one study was available for caries incidence and this related to children, providing low quality evidence that the addition of arginine to fluoride toothpaste had any effect. Three studies in children considered caries progression and the one meta-analysis that was conducted suggested a beneficial effect from the addition of arginine, however, the authors highlight a number of concerns related to these studies. Most of them were only of six-months' duration and QLF was used to measure caries progression. In addition, there were no pre-published trial protocols and poor descriptions of the randomisation process. Employees of commercial companies were involved in the included studies.

The review's findings are similar to a 2015 review by Li et al. ${ }^{1}$ which included more studies (ten), although they again considered the available evidence to be low, noting that they were all industry sponsored.

The current evidence for arginine is of low quality and currently arginine toothpaste is about $40 \%$ more expensive than conventional toothpaste. At present there is no reason to recommend arginine-fluoride toothpaste in preference to conventional fluoride toothpaste, and high quality well designed and reported studies of longer duration are needed to clarify any potential caries preventive effect.

Derek Richards

Department of Public Health, NHS Forth Valley and Centre for Evidence-based Dentistry, Dental Health Services Research Unit, Dundee Dental Hospital and School Dundee University, Dundee, Scotland

1. Li J, Huang Z, Mei L, Li G, Li H. Anti-caries effect of arginine-containing formulations in vivo: A systematic review and meta-analysis. Caries Res 2015; 49: 606-617.

Evidence-Based Dentistry (2017) 18, 71. doi:10.1038/sj.ebd.6401251 